\title{
Robust Coordinated Control Algorithm for Multiple Marine Vessels with External Disturbances
}

\author{
Weixue Liu, ${ }^{1}$ Jianfang Jiao, ${ }^{1}$ Hamid Reza Karimi, ${ }^{2}$ and Jian Jiao ${ }^{3}$ \\ ${ }^{1}$ Bohai University, Jinzhou 121013, China \\ ${ }^{2}$ Department of Engineering, Faculty of Engineering and Science, University of Agder, 4898 Grimstad, Norway \\ ${ }^{3}$ No. 803 Research Institute of Shanghai Academy of Spaceflight Technology, No. 710 Yishan Road, Shanghai 200233, China
}

Correspondence should be addressed to Jianfang Jiao; jiaojianfangheu@163.com

Received 16 August 2013; Accepted 23 September 2013

Academic Editor: Zhiguang Feng

Copyright (C) 2013 Weixue Liu et al. This is an open access article distributed under the Creative Commons Attribution License, which permits unrestricted use, distribution, and reproduction in any medium, provided the original work is properly cited.

\begin{abstract}
The problem of coordinated control for multiple marine vessels in the presence of external disturbances is considered in this paper. A robust coordinated control algorithm is proposed for multiple marine vessels. The proposed robust coordinated control algorithm is divided into two parts. The first part develops an extended state observer to estimate the disturbances of marine vessels. The second part presents a robust coordinated control algorithm based on the output of the extended state observer. Furthermore, the robust coordinated control algorithm is designed using the dynamic surface control method. In light of the leader-follower strategy, the trajectory for each vessel is defined according to the desired trajectory of the assigned leader and the relative distance with respect to the leader. The effectiveness of the proposed coordination algorithm is demonstrated by the simulation results.
\end{abstract}

\section{Introduction}

In recent years, coordinated control of multiple vehicles has received increasing attention as an emerging technology [1]. Multiple vehicles can perform many complex tasks effectively with less time and lower cost than a single vehicle. And multiple vehicles can accomplish some tasks which cannot be executable by a single one. In order to perform these complicated practical tasks, it is necessary for these vehicles to move collectively as a whole formation. In practice, many relevant applications of coordinated control can be found on the land, in the sea, and in the air [2]. For instance, in the operations of underway replenishment by a fleet of surface vessels, it is required that the replenished vehicle should maintain a fixed relative position with respect to the replenishing one, in order to ensure the replenishment operation performed safely and effectively.

The problem of coordinated formation control has been reported in a large number of recent publications. Basic approaches of the coordinated control include leaderfollower approach [3-5], behavioral approach $[6,7]$, and virtual structures approach $[8,9]$. In the leader-follower approach, some agents are considered as the leaders, and the rest ones are considered as the followers. The followers will track the leaders, and the leaders will track the predefined desired trajectories. This method is easy to be manipulated and implemented. However, the main criticism of the leaderfollower approach is that it depends heavily on the leader to achieve the goal of the formation task which may be undesirable [3-5]. In the behavioral approach, the collision avoidance/obstacle avoidance and the target tracking are prescribed for each agent, and the whole formation is achieved by calculating the weight of the relative importance of each behavior. However, it is difficult to analyze the stability of the group behavior using such approach $[6,7]$. In the virtual-structure approach, each member in the formation is considered as a particle embedded in a rigid geometric structure, but the relative applications are limited when the formation structure is time-varying or needs to be frequently reconfigured $[8,9]$.

Some advanced approaches including graph theory [10], passivity-based control [11, 12], and hybrid control [13] are also used for coordinated control of multiple marine vessels. Most results about the coordinated control problem addressed in the earlier papers are on the assumption that marine vessels are free from environmental disturbances. 
However, coordinated control for multiple surface vessels encountering exogenous disturbances adds a new level of complexity to the problem. Other advanced methods are proposed to solve the robust coordinated formation control problem, for example, the Lagrangian approach [14], the nonlinear model predictive control [15], the adaptive control [16], and the sliding mode control [17]. In addition, the fault tolerant control and the fault diagnosis are studied in references [18-20]. In particular, the problem of coordinated path following multiple vessels has also been discussed in the related literature studies [21, 22]. The robustness to environmental disturbances is highly important when performing practical marine and offshore tasks for surface vessels, which is also the concerned issue in this paper. The core of the extended state observer is that the disturbances and the unknown dynamics can be considered as extend state, and then the detailed values can be estimated by designed observer. The correlative applications can be found in literature studies [23-26]. The stability of the extended state observer is analyzed in [27-29]. The robust coordination control algorithm for multiple surface vessels based on extend state observer and robust control technology is studied in this paper. The designed controller is useful for the practical marine operations.

In this paper, we consider the problem of coordinated formation control of multiple surface vessels in the presence of exogenous disturbances. The coordinated formation controller is proposed by combining the extended state observer and dynamic surface control using the leaderfollower strategy. The extended state observer is developed to estimate the external disturbances of the surface vessels. The coordinated control algorithm is accomplished based on the output of the extended state observer. Furthermore, the trajectory of each vessel is defined using the desired trajectory of the assigned leader and the relative distance with respect to the leader. This paper is organized as follows. In Section 2, the vessel model is established. Section 3 contains a detailed algorithm of the coordination formation control for multiple vessels. Simulation is carried out in Section 4, and we draw conclusions in Section 5.

\section{Preliminaries}

The vessel model can be divided into two parts: the kinematics and nonlinear dynamics. Generally, only the motion in the horizontal plane is considered for the surface vessel. The elements corresponding to heave, roll, and pitch are neglected. The dynamic model for the ith surface vessel can be represented by the following 3 degrees of freedom (DOF) [30]:

$$
\begin{gathered}
\dot{\eta}_{i}=\mathbf{R}_{i}\left(\psi_{i}\right) \mathbf{v}_{i} \\
\mathbf{M}_{i} \dot{\mathbf{v}}_{i}+\mathrm{C}_{i}\left(\mathbf{v}_{i}\right) \mathbf{v}_{i}+\mathbf{D}_{i}\left(\mathbf{v}_{i}\right) \mathbf{v}_{i}=\tau_{i}+\tau_{d i}
\end{gathered}
$$

where $\eta_{i}=\left[n_{i}, e_{i}, \psi_{i}\right]^{T}$ denotes the north position, east position, and orientation which are decomposed in the Earthfixed reference frame, $\mathbf{v}_{i}=\left[u_{i}, v_{i}, r_{i}\right]^{T}$ denotes the linear surge velocity, sway velocity, and angular velocity which are decomposed in the body-fixed reference frame. $\mathbf{R}_{i}\left(\psi_{i}\right)$ is the transformations matrix from the body-fixed reference frame to the Earth-fixed reference frame, the form of which is as follows:

$$
\mathbf{R}_{i}\left(\psi_{i}\right)=\left[\begin{array}{ccc}
\cos \left(\psi_{i}\right) & -\sin \left(\psi_{i}\right) & 0 \\
\sin \left(\psi_{i}\right) & \cos \left(\psi_{i}\right) & 0 \\
0 & 0 & 1
\end{array}\right]
$$

The transformations matrix satisfies $\mathbf{R}_{i}^{-1}\left(\psi_{i}\right)=\mathbf{R}_{i}^{T}\left(\psi_{i}\right)$, for all $\psi_{i} \cdot \mathbf{M}_{i}$ denotes the system inertia mass matrix including added mass which is positive definite. $\mathbf{C}_{i}\left(\mathbf{v}_{i}\right)$ and $\mathbf{D}_{i}\left(\mathbf{v}_{i}\right)$ denote the Coriolis-centripetal matrix and damping matrix, respectively. The detailed representation of the above three system matrices can be found in reference [30]. $\tau_{i}=$ $\left[\begin{array}{lll}\tau_{u i} & \tau_{v i} & \tau_{r i}\end{array}\right]^{T}$ is the vector of forces and torques input from the thruster system. $\tau_{d i}$ is the vector of external environment forces and torques input which is generated by wind, wave, and current.

In order to design the backstepping sliding mode controller, we transform the vessel model as follows:

$$
\mathbf{v}_{i}=\mathbf{R}_{i}^{-1}\left(\psi_{i}\right) \dot{\eta}_{i}
$$

because $\dot{\mathbf{R}}_{i}\left(\psi_{i}\right)=\mathbf{R}_{i}\left(\psi_{i}\right) S$, where

$$
S=\left[\begin{array}{ccc}
0 & -r_{i} & 0 \\
r_{i} & 0 & 0 \\
0 & 0 & 0
\end{array}\right]=-S^{T}
$$

with $r_{i}$ being the angular velocity in the body-fixed reference frame. We can obtain that

$$
\begin{aligned}
\dot{\mathbf{v}}_{i} & =\dot{\mathbf{R}}_{i}^{-1}\left(\psi_{i}\right) \dot{\eta}_{i}+\mathbf{R}_{i}^{-1}\left(\psi_{i}\right) \ddot{\eta}_{i} \\
& =-\mathbf{R}_{i}^{-1}\left(\psi_{i}\right) \dot{\mathbf{R}}_{i}\left(\psi_{i}\right) \mathbf{R}_{i}^{-1}\left(\psi_{i}\right) \dot{\eta}_{i}+\mathbf{R}_{i}^{-1}\left(\psi_{i}\right) \ddot{\eta}_{i} .
\end{aligned}
$$

Taking (4) and (6) into the vessel dynamic model (2) yields

$$
\begin{aligned}
& \mathbf{M}_{i} \mathbf{R}_{i}^{-1}\left(\psi_{i}\right) \ddot{\eta}_{i}-\mathbf{M}_{i} \mathbf{R}_{i}^{-1}\left(\psi_{i}\right) \dot{\mathbf{R}}_{i}\left(\psi_{i}\right) \mathbf{R}_{i}^{-1}\left(\psi_{i}\right) \dot{\eta}_{i} \\
& \quad+\mathbf{C}_{i}\left(\mathbf{v}_{i}\right) \mathbf{R}_{i}^{-1}\left(\psi_{i}\right) \dot{\eta}_{i}+\mathbf{D}_{i}\left(\mathbf{v}_{i}\right) \mathbf{R}_{i}^{-1}\left(\psi_{i}\right) \dot{\eta}_{i}=\tau_{i}+\tau_{d i}
\end{aligned}
$$

The above equation can be written as

$$
\mathbf{M}_{n i}\left(\eta_{i}\right) \ddot{\eta}_{i}+\mathbf{C}_{n i}\left(\eta_{i}, \dot{\eta}_{i}\right) \dot{\eta}_{i}+\mathbf{D}_{n i}\left(\eta_{i}, \dot{\eta}_{i}\right) \dot{\eta}_{i}=\tau_{i}+\tau_{d i},
$$

where

$$
\begin{gathered}
\mathbf{M}_{n i}\left(\eta_{i}\right)=\mathbf{M}_{i} \mathbf{R}_{i}^{-1}\left(\psi_{i}\right) ; \\
\mathbf{C}_{n i}\left(\eta_{i}, \dot{\eta}_{i}\right)=\left[\mathbf{C}_{i}\left(\mathbf{v}_{i}\right)-\mathbf{M}_{i} \mathbf{R}_{i}^{-1}\left(\psi_{i}\right) \dot{\mathbf{R}}_{i}\left(\psi_{i}\right)\right] \mathbf{R}_{i}^{-1}\left(\psi_{i}\right) ; \\
\mathbf{D}_{n i}\left(\eta_{i}, \dot{\eta}_{i}\right)=\mathbf{D}_{i}\left(\mathbf{v}_{i}\right) \mathbf{R}_{i}^{-1}\left(\psi_{i}\right) .
\end{gathered}
$$

\section{Coordinated Formation Controller Design}

In this section, the controller is designed from two inspects. One is the extended state observer design for each vessel, and the other is the coordinated controller for multiple vessels based on the output of the extended state observer. 
3.1. Extended State Observer Design. In this section, we design the extended state observer for each vessel to estimate the disturbances.

Let $\dot{\eta}_{i}=\mathbf{v}_{n i}$, and then (8) can be written as

$$
\mathbf{M}_{n i}\left(\eta_{i}\right) \dot{\mathbf{v}}_{n i}+\mathbf{C}_{n i}\left(\eta_{i}, \mathbf{v}_{n i}\right) \mathbf{v}_{n i}+\mathbf{D}_{n i}\left(\eta_{i}, \mathbf{v}_{n i}\right) \mathbf{v}_{n i}=\tau_{i}+\tau_{d i} .
$$

Then we have

$$
\begin{aligned}
& \dot{\eta}_{i}=\mathbf{v}_{n i}, \\
& \dot{\mathbf{v}}_{n i}=\mathbf{M}_{n i}^{-1}\left(\eta_{i}\right) \tau_{i} \\
& +\mathbf{M}_{n i}^{-1}\left(\eta_{i}\right)\left(-\mathbf{C}_{n i}\left(\eta_{i}, \mathbf{v}_{n i}\right) \mathbf{v}_{n i}-\mathbf{D}_{n i}\left(\eta_{i}, \mathbf{v}_{n i}\right) \mathbf{v}_{n i}+\tau_{d i}\right) .
\end{aligned}
$$

Let $\mathbf{K}_{i}=\mathbf{M}_{n i}^{-1}\left(\eta_{i}\right)\left(-\mathbf{C}_{n i}\left(\eta_{i}, \mathbf{v}_{n i}\right) \mathbf{v}_{n i}-\mathbf{D}_{n i}\left(\eta_{i}, \mathbf{v}_{n i}\right) \mathbf{v}_{n i}+\right.$ $\left.\tau_{d i}\right), \mathbf{u}_{i}=\mathbf{M}_{n i}^{-1}\left(\eta_{i}\right) \tau_{i}$. Then the above equation can be rewritten as

$$
\begin{gathered}
\dot{\eta}_{i}=\mathbf{v}_{n i}, \\
\dot{\mathbf{v}}_{n i}=\mathbf{K}_{i}+\mathbf{u}_{i} .
\end{gathered}
$$

Here $\mathbf{K}_{i}$ is assumed to be unknown. We assume that $\mathbf{K}_{i}$ is an extended state. However, $\mathbf{K}_{i}$ can be estimated using an extended state observer. Then the disturbances are observed and compensated by the designed controller.

The extended state observer is designed as

$$
\begin{gathered}
\mathbf{z}_{1 \mathrm{i}}=\eta_{i}-\widehat{\eta}_{i}, \\
\dot{\hat{\eta}}_{i}=\widehat{\mathbf{v}}_{n i}+\beta_{1} \operatorname{fal}_{1}\left(\mathbf{z}_{1 i}, \alpha, \delta\right), \\
\dot{\widehat{\mathbf{v}}}_{n i}=\widehat{\mathbf{K}}_{i}+\beta_{2} \operatorname{fal}_{2}\left(\mathbf{z}_{1 i}, \alpha, \delta\right)+\mathbf{u}_{i}, \\
\dot{\widehat{\mathbf{K}}}_{i}=\beta_{3} \operatorname{fal}_{3}\left(\mathbf{z}_{1 i}, \alpha, \delta\right),
\end{gathered}
$$

where

$$
\operatorname{fal}\left(\mathbf{z}_{1 i}, \alpha, \delta\right)= \begin{cases}\left|\mathbf{z}_{1 i}\right|^{\alpha} \operatorname{sign}\left(\mathbf{z}_{1 i}\right), & \left|\mathbf{z}_{1 i}\right|>\delta, \\ \frac{\mathbf{z}_{1 i}}{\delta^{\alpha-1}}, & \left|\mathbf{z}_{1 i}\right| \leq \delta .\end{cases}
$$

And $\delta>0,0<\alpha<1$.

Set $\mathbf{z}_{1 i}=\eta_{i}-\widehat{\eta}_{i}, \mathbf{z}_{2 i}=\mathbf{v}_{n i}-\widehat{\mathbf{v}}_{n i}, \mathbf{z}_{3 i}=\mathbf{K}_{i}-\widehat{\mathbf{K}}_{i}, \widehat{\eta}_{i}, \widehat{\mathbf{v}}_{n i}, \widehat{\mathbf{K}}_{i}$ are the estimated values of $\eta_{i}, \mathbf{v}_{n i}, \mathbf{K}_{i}$, respectively. Taking the derivative of $\mathbf{z}_{1 i}, \mathbf{z}_{2 i}, \mathbf{z}_{3 i}$, respectively, we can obtain that

$$
\begin{aligned}
\dot{\mathbf{z}}_{1 i} & =\widehat{\mathbf{v}}_{n i}+\mathbf{z}_{2 i}-\widehat{\mathbf{v}}_{n i}-\beta_{1} \mathrm{fal}_{1}\left(\mathbf{e}_{i}, \alpha, \delta\right) \\
& =\mathbf{z}_{2 i}-\beta_{1} \mathrm{fal}_{1}\left(\mathbf{z}_{1 i}, \alpha, \delta\right) \\
\dot{\mathbf{z}}_{2 i} & =\mathbf{K}_{i}+\mathbf{u}_{i}-\widehat{\mathbf{K}}_{i}-\beta_{2} \mathrm{fal}_{2}\left(\mathbf{z}_{1 i}, \alpha, \delta\right)-\mathbf{u}_{i} \\
& =\mathbf{z}_{3 i}-\beta_{2} \mathrm{fal}_{2}\left(\mathbf{z}_{1 i}, \alpha, \delta\right) \\
\dot{\mathbf{z}}_{3 i} & =\dot{\mathbf{K}}_{i}-\beta_{3} \mathrm{fal}_{3}\left(\mathbf{z}_{1 i}, \alpha, \delta\right) .
\end{aligned}
$$

The following assumptions are presumed.

(1) The possibly unknown function $\mathbf{K}_{i}$ is continuously differentiable with respect to their variables. $\left|\dot{\mathbf{K}}_{i}\right| \leq M$ for all $t>0$, where $M$ is a positive constant.

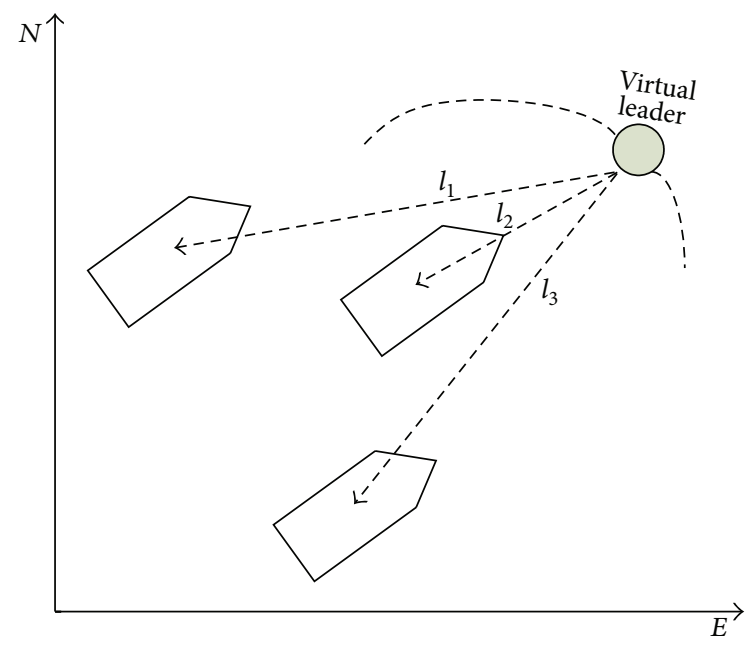

FIGURE 1: The formation of these vessels.

(2) Let $\mathbf{z}=\left[\mathbf{z}_{1}, \mathbf{z}_{2}, \mathbf{z}_{3}\right]^{T}$, and $\|\bullet\|$ denotes the Euclid norm of $\mathbb{R}^{9}$. There exist positive definite constants $\lambda_{i}(i=$ $1,2,3,4), \alpha$ and continuous differentiable functions $V, W: \mathbb{R}^{9} \rightarrow \mathbb{R}$ such that

$$
\begin{aligned}
\text { (i) } & \lambda_{1}\|\mathbf{z}\|^{2} \leq \mathbf{V}(\mathbf{z}) \leq \lambda_{2}\|\mathbf{z}\|^{2}, \lambda_{3}\|\mathbf{z}\|^{2} \leq \mathbf{W}(\mathbf{z}) \leq \\
& \lambda_{4}\|\mathbf{z}\|^{2}, \\
\text { (ii) } & \left(\partial V / \partial \mathbf{z}_{1}\right)\left(\mathbf{z}_{2}-f_{1}\left(\mathbf{z}_{1}\right)\right)+\left(\partial V / \partial \mathbf{z}_{2}\right)\left(\mathbf{z}_{2}-f_{2}\left(\mathbf{z}_{1}\right)\right)+ \\
& \left(\partial V / \partial \mathbf{z}_{3}\right) f_{3}\left(\mathbf{z}_{1}\right) \leq-W(\mathbf{z}), \\
\text { (iii) } & \left\|\partial V / \partial \mathbf{z}_{3}\right\| \leq \alpha\|\mathbf{z}\| .
\end{aligned}
$$

The stability for the extended state observer is analyzed in [24]. Then we can obtain $\widehat{\eta}_{i} \rightarrow \eta_{i}, \widehat{\mathbf{v}}_{n i} \rightarrow \mathbf{v}_{n i}, \widehat{\mathbf{K}}_{i} \rightarrow \mathbf{K}_{i}$.

\subsection{Coordinated Controller Design}

3.2.1. Formation Setup. This paper considers a fleet of $n$ vessels to perform the desired coordination formation task. Each vessel in the formation is identified by the index set $I=[1,2, \ldots, n]$. The desired formation is established using the leader-follower strategy as shown in Figure 1. Furthermore, the leader is a virtual vessel. If we assume the desired trajectory of the leader vessel is denoted as $\eta_{d}$, where $\eta_{d}=\left[n_{d}(t), e_{d}(t), \psi_{d}(t)\right]^{T}, n_{d}(t), e_{d}(t)$ are sufficiently smooth functions, and $\psi_{d}(t)=\arctan \left(\dot{e}_{d}(t) / \dot{n}_{d}(t)\right)$. That means the vessel direction is chosen as the tangential vector of the respective desired trajectory. If we define the relative distance between the follower vessel and the leader vessel as $\mathbf{l}_{i}=\left[x_{0 i}, y_{0 i}, \psi_{0 i}\right]^{T}$, then the desired trajectory of the follower vessel is denoted as $\eta_{d i}=\eta_{d}+\mathbf{R}\left(\psi_{d}\right) \mathbf{l}_{i}$.

3.2.2. Controller Design. Define the first dynamic surface as

$$
\mathbf{S}_{1 i}=\widehat{\eta}_{i}-\eta_{d i} .
$$

Taking the derivative of the first surface, with (13), we obtain

$$
\begin{aligned}
\dot{\mathbf{S}}_{1 i} & =\dot{\hat{\eta}}_{i}-\dot{\eta}_{d i} \\
& =\widehat{\mathbf{v}}_{n i}+\beta_{1} \operatorname{fal}_{1}\left(\mathbf{z}_{1 i}, \alpha, \delta\right)-\dot{\eta}_{d i} .
\end{aligned}
$$


Define a virtual velocity $\mathbf{v}_{r i}$ as follows:

$$
\mathbf{v}_{r i}=\dot{\eta}_{d i}-\beta_{1} \mathrm{fal}_{1}\left(\mathbf{z}_{1 i}, \alpha, \delta\right)-\boldsymbol{\Lambda}_{1} \mathbf{S}_{1 i}
$$

where $\Lambda_{1}$ is a positive definite matrix. With this definition, if $\mathbf{v}_{r i}=\widehat{\mathbf{v}}_{n i}$, then $\dot{\mathbf{S}}_{1 i}=-\boldsymbol{\Lambda}_{1} \mathbf{S}_{1 i}$. Then $\mathbf{S}_{1 i} \rightarrow 0$ with a convergence rate determined by the choice of $\Lambda_{1}$. Because of the definition of $\mathbf{S}_{1 i}$, this will also guarantee that $\hat{\eta}_{i} \rightarrow \eta_{i} \rightarrow$ $\eta_{d i}$.

$\mathbf{v}_{r i}$ is passed through a first order filter in order to avoid the problem existed in the backstepping scheme:

$$
\mathbf{T} \dot{\mathbf{v}}_{d i}+\mathbf{v}_{d i}=\mathbf{v}_{r i}
$$

where $\mathbf{T}$ is a diagonal matrix of the filter time constants which are chosen to be as small as possible. Because $\widehat{\mathbf{v}}_{n i} \rightarrow \mathbf{v}_{n i}$, then we can define the second sliding surface as

$$
\mathbf{S}_{2 i}=\widehat{\mathbf{v}}_{n i}-\mathbf{v}_{d i},
$$

where $\mathbf{v}_{d i}$ is the estimated value of $\mathbf{v}_{r i}$; take the derivative of $\mathbf{v}_{d i}$, then we have

$$
\dot{\mathbf{v}}_{d i}=\mathbf{T}^{-1}\left(\mathbf{v}_{r i}-\mathbf{v}_{d i}\right) .
$$

Taking the derivative of the second surface, with (13), we have

$$
\begin{aligned}
\dot{\mathbf{S}}_{2 i} & =\dot{\hat{\mathbf{v}}}_{n i}-\dot{\mathbf{v}}_{d i} \\
& =\widehat{\mathbf{K}}_{i}+\beta_{2} \mathrm{fal}_{2}\left(\mathbf{z}_{1 i}, \alpha, \delta\right)+\mathbf{u}_{i}-\dot{\mathbf{v}}_{d i} .
\end{aligned}
$$

We consider the following Lyapunov function candidate:

$$
\mathbf{V}_{1 i}=\frac{1}{2} \mathbf{S}_{2 i}^{T} \mathbf{S}_{2 i}
$$

We take the time derivative of (25):

$$
\dot{\mathbf{V}}_{1 i}=\mathbf{S}_{2 i}^{T}\left(\widehat{\mathbf{K}}_{i}+\beta_{2} \mathrm{fal}_{2}\left(\mathbf{z}_{1 i}, \alpha, \delta\right)+\mathbf{u}_{i}-\dot{\mathbf{v}}_{d i}\right) .
$$

So we choose the control input as $\mathbf{u}_{i}=\dot{\mathbf{v}}_{d i}-\widehat{\mathbf{K}}_{i}-\beta_{2} \mathrm{fal}_{2}\left(\mathbf{z}_{1 i}\right.$, $\alpha, \delta)-\mathbf{K}_{D} \mathbf{S}_{2 i}$.

The control input of the vessel is $\tau_{i}=\mathbf{M}_{n i}\left(\eta_{i}\right) \mathbf{u}_{i}$. So the control force input $\tau$ is selected as

$$
\tau_{i}=\mathbf{M}_{n i}\left(\eta_{i}\right)\left(\dot{\mathbf{v}}_{d i}-\widehat{\mathbf{K}}_{i}-\beta_{2} \mathrm{fal}_{2}\left(\mathbf{z}_{1 i}, \alpha, \delta\right)-\mathbf{K}_{D} \mathbf{S}_{2 i}\right) .
$$

Theorem 1. Consider the vessel with the nonlinear model as in (1), (2), and (8), with the control law (27), and then one can guarantee that the vessels approach the desired trajectory ultimately while holding the desired formation structure.

Proof. With the definition of the second surface, (19) can be rewrite as

$$
\dot{\mathbf{S}}_{1 i}=\mathbf{S}_{2 i}+\mathbf{v}_{d i}-\beta_{1} \mathrm{fal}_{1}\left(\mathbf{z}_{1 i}, \alpha, \delta\right)-\dot{\eta}_{d i}
$$

Define the estimated error of the first order filter as

$$
\mathbf{S}_{3 i}=-\mathbf{T} \dot{\mathbf{v}}_{d i}=\mathbf{v}_{d i}-\mathbf{v}_{r i} .
$$

Taking the derivative of $\mathbf{S}_{3 i}$ yields

$$
\begin{aligned}
\dot{\mathbf{S}}_{3 i} & =\dot{\mathbf{v}}_{d i}-\mathbf{v}_{r i} \\
& =\dot{\mathbf{v}}_{d i}+\beta_{1} \frac{d \mathrm{fal}_{1}\left(\mathbf{z}_{1 i}, \alpha, \delta\right)}{d t}+\boldsymbol{\Lambda}_{1} \dot{\mathbf{S}}_{1 i}-\ddot{\eta}_{d i} \\
& =-\frac{\mathbf{S}_{3 i}}{\mathbf{T}}+\beta_{1} \frac{d \mathrm{fal}_{1}\left(\mathbf{z}_{1 i}, \alpha, \delta\right)}{d t}+\Lambda_{1} \dot{\mathbf{S}}_{1 i}-\ddot{\eta}_{d i} \\
& =-\frac{\mathbf{S}_{3 i}}{\mathbf{T}}+g\left(\beta_{1}, \mathbf{z}_{1 i}, \alpha, \delta, \dot{\mathbf{z}}_{1 i}, \ddot{\eta}_{d i}, \dot{\mathbf{S}}_{1 i}\right) .
\end{aligned}
$$

With (19) and (21), we obtain

$$
\dot{\mathbf{S}}_{1 i}=\mathbf{S}_{2 i}+\mathbf{S}_{3 i}-\Lambda_{1 i} \mathbf{S}_{1 i} .
$$

Define the Lyapunov function as

$$
\mathbf{V}_{i}=\frac{1}{2} \mathbf{S}_{1 i}^{T} \mathbf{S}_{1 i}+\frac{1}{2} \mathbf{S}_{2 i}^{T} \mathbf{S}_{2 i}+\frac{1}{2} \mathbf{S}_{3 i}^{T} \mathbf{S}_{3 i} .
$$

Differentiating the above equation yields

$$
\begin{aligned}
\dot{\mathbf{V}}_{i}= & \mathbf{S}_{1 i}^{T} \dot{\mathbf{S}}_{1 i}+\mathbf{S}_{2 i}^{T} \dot{\mathbf{S}}_{2 i}+\mathbf{S}_{3 i}^{T} \dot{\mathbf{S}}_{3 i} \\
= & \mathbf{S}_{1 i}^{T}\left(\mathbf{S}_{2 i}+\mathbf{S}_{3 i}-\mathbf{\Lambda}_{1} \mathbf{S}_{1 i}\right)-\mathbf{S}_{2 i}^{T} \mathbf{K}_{D} \mathbf{S}_{2 i}+\mathbf{S}_{3 i}^{T} \\
& \times\left(-\frac{\mathbf{S}_{3 i}}{\mathbf{T}}+g\left(\beta_{1}, \mathbf{z}_{1 i}, \alpha, \delta, \dot{\mathbf{z}}_{1 i}, \ddot{\eta}_{d i}, \dot{\mathbf{S}}_{1 i}\right)\right) \\
= & -\mathbf{S}_{1 i}^{T} \boldsymbol{\Lambda}_{1} \mathbf{S}_{1 i}+\mathbf{S}_{1 i}^{T} \mathbf{S}_{2 i}+\mathbf{S}_{1 i}^{T} \mathbf{S}_{3 i} \\
& -\mathbf{S}_{2 i}^{T} \mathbf{K}_{D} \mathbf{S}_{2 i}-\frac{\mathbf{S}_{3 i}^{T} \mathbf{S}_{3 i}}{\mathbf{T}} \\
& +\mathbf{S}_{3 i}^{T} g\left(\beta_{1}, \mathbf{z}_{1 i}, \alpha, \delta, \dot{\mathbf{z}}_{1 i}, \ddot{\eta}_{d i}, \dot{\mathbf{S}}_{1 i}\right) .
\end{aligned}
$$

If we define the maximum of $g\left(\beta_{1}, \mathbf{z}_{1 i}, \alpha, \delta, \dot{\mathbf{z}}_{1 i}, \ddot{\eta}_{d i}, \dot{\mathbf{S}}_{1 i}\right)$ is $g_{\text {max }}$, we can know that $\mathbf{S}_{1 i}^{T} \mathbf{S}_{1 i}+\mathbf{S}_{2 i}^{T} \mathbf{M S}_{2 i}+\mathbf{S}_{3 i}^{T} \mathbf{S}_{3 i} \leq 2 p, p$ is a positive constant. Then we can obtain that $V_{i} \leq p$. Let $\Lambda_{1}=\mathbf{K}_{D}=\alpha_{0}+2 I_{3}$, and the filter time constant can be chosen as $\mathbf{T}=\left(I_{3}+\left(g_{\max }^{T} g_{\max } / 2 \varepsilon I_{3}\right)+\alpha_{0}\right)^{-1}$; then we can obtain that

$$
\begin{aligned}
\dot{\mathbf{V}}_{i}= & -\mathbf{S}_{1 i}^{T}\left(\alpha_{0}+2 I_{3}\right) \mathbf{S}_{1 i}-\mathbf{S}_{2 i}^{T}\left(\alpha_{0}+2 I_{3}\right) \mathbf{S}_{2 i} \\
& -\mathbf{S}_{3 i}^{T}\left(I_{3}+\left(\frac{g_{\max }^{2}}{2 \varepsilon I_{3}}\right)+\alpha_{0}\right) \mathbf{S}_{3 i} \\
& +\mathbf{S}_{1 i}^{T} \mathbf{S}_{2 i}+\mathbf{S}_{1 i}^{T} \mathbf{S}_{3 i}+\mathbf{S}_{3 i}^{T} g \\
\leq & -\mathbf{S}_{1 i}^{T}\left(\alpha_{0}+2 I_{3}\right) \mathbf{S}_{1 i}-\mathbf{S}_{2 i}^{T}\left(\alpha_{0}+2 I_{3}\right) \mathbf{S}_{2 i} \\
& +\frac{2 \mathbf{S}_{1 i}^{T} \mathbf{S}_{1 i}+\mathbf{S}_{2 i}^{T} \mathbf{S}_{2 i}+\mathbf{S}_{3 i}^{T} \mathbf{S}_{3 i}}{2} \\
& -\mathbf{S}_{3 i}^{T} \mathbf{S}_{3 i}-\mathbf{S}_{3 i}^{T} \alpha_{0} \mathbf{S}_{3 i}-\mathbf{S}_{3 i}^{T}\left(\frac{g_{\max }^{T} g_{\max }}{2 \varepsilon I_{3}}\right) \mathbf{S}_{3 i} \\
& +\frac{g_{\max }^{2} \mathbf{S}_{3 i}^{T} \mathbf{S}_{3 i}}{2 \varepsilon I_{3}} \frac{g^{T} g}{g_{\max }^{2}}+\frac{\varepsilon}{2} \\
\leq & -2 \alpha_{0} \mathbf{V}_{i}+\frac{\varepsilon}{2} .
\end{aligned}
$$




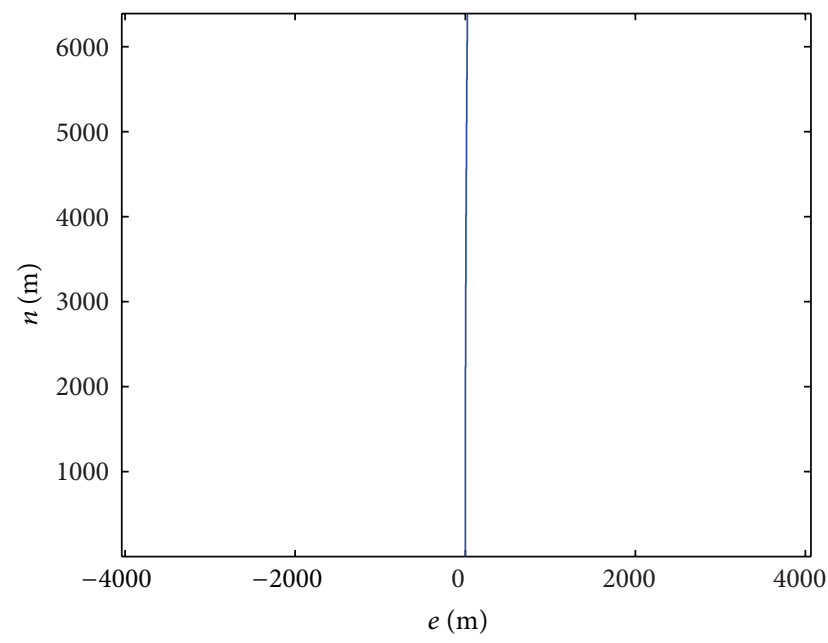

Figure 2: The movement of the vessel in the plane.
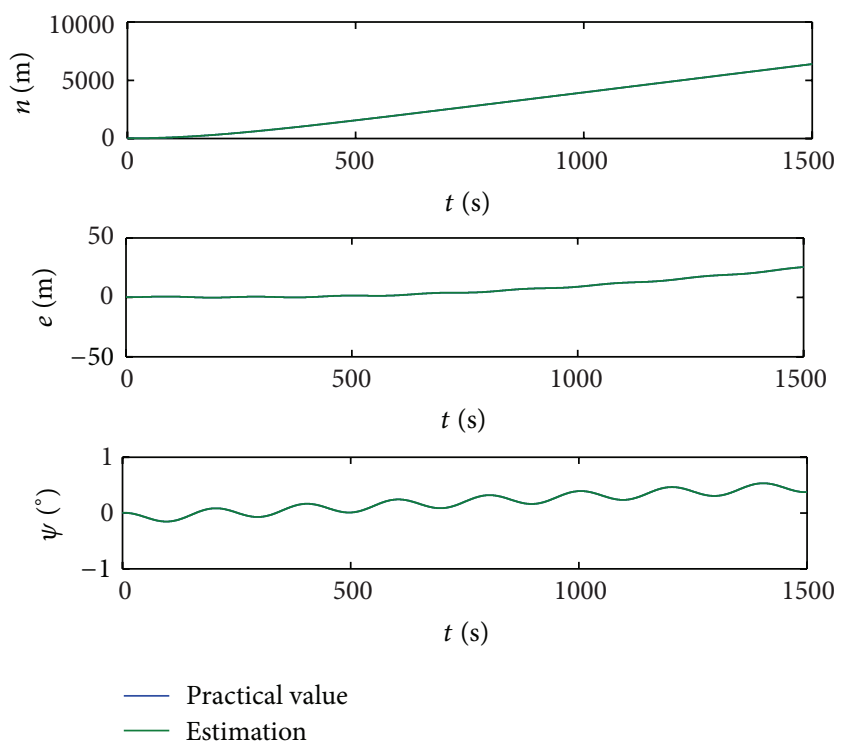

Figure 3: The practical value and estimated value of the positions.

If we choose $\alpha_{0}>\varepsilon / 2 p$, then $\dot{V}_{i}<0$. We can guarantee that $\mathbf{S}_{2 i} \rightarrow 0$. This implies $\widehat{\mathbf{v}}_{n i} \rightarrow \mathbf{v}_{n i} \rightarrow \mathbf{v}_{d i}$, in turn, $\mathbf{S}_{1 i} \rightarrow 0$ and $\hat{\eta}_{i} \rightarrow \eta_{i} \rightarrow \eta_{d i}$.

\section{Simulation Results}

In this section, experimental simulations are carried out to evaluate the effectiveness of the proposed coordinated formation control algorithm. The detailed parameters of the vessel are presented in the literature [11]. At the beginning, the proposed extended state observer of one vessel is evaluated by the simulation. Similarly, the performance of the extended state observer of other vessels is achieved. Compare with the existing literature studies, we let the initial position of the vessel is $\eta=[0,0,0]^{T}$. The vessel moves in a beeline northward. assume that the vessels encounter the wind, wave, and current. The wind is assumed to be fixed direction and
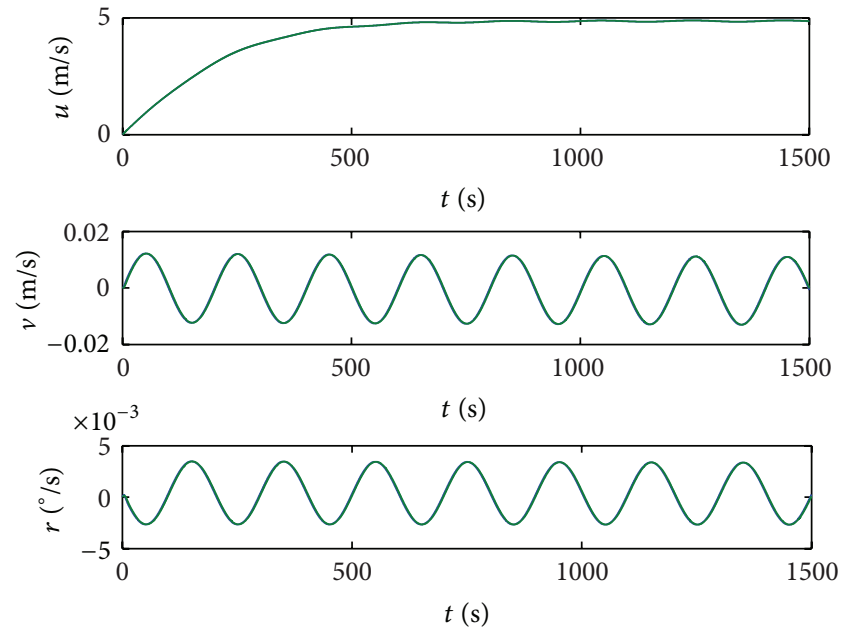

- Practical value

Estimation

FIGURE 4: The practical value and estimated value of the velocities.
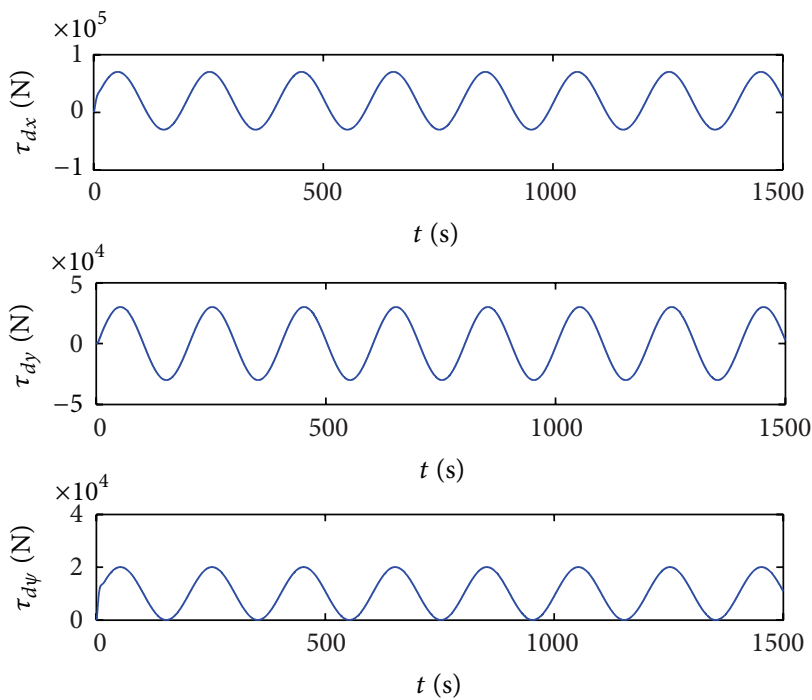

FIGURE 5: The estimated value of the disturbances.

fixed velocity, and then the disturbance of wind is a constant; the wave and current are assumed to be the sine wave with a fixed frequency at one time. The external disturbances can be chosen as

$$
\begin{aligned}
\tau_{d}=10^{6} *[ & 0.05 \sin (\pi t / 100)+0.02, \\
& 0.03 \sin (\pi t / 100), \\
& 0.01 \sin (\pi t / 100)+0.01]^{T}(N) .
\end{aligned}
$$

In the simulation, we assume that the external disturbances are unknown. The proposed observer parameters are selected as $\beta_{1}=30, \beta_{2}=15, \beta_{3}=5, \alpha=0.25$, and $\delta=0.1$.

The simulation results are shown in Figures 2 to 5 . Figure 2 shows the movements for the vessel in the plane. The practical value and estimated value of the north position, east 


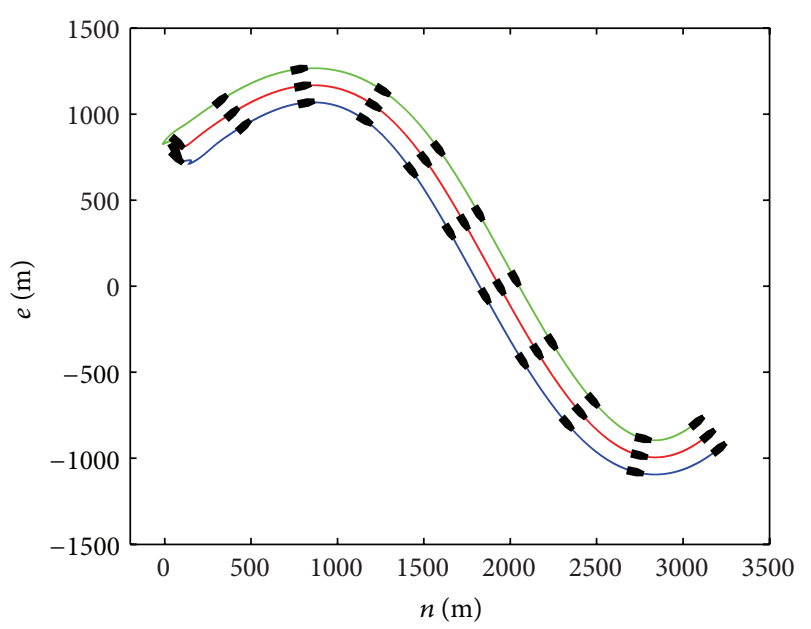

- Vessel 1
- Vessel 2
- Vessel 3

Figure 6: Coordinated trajectory tracking of the vessels.

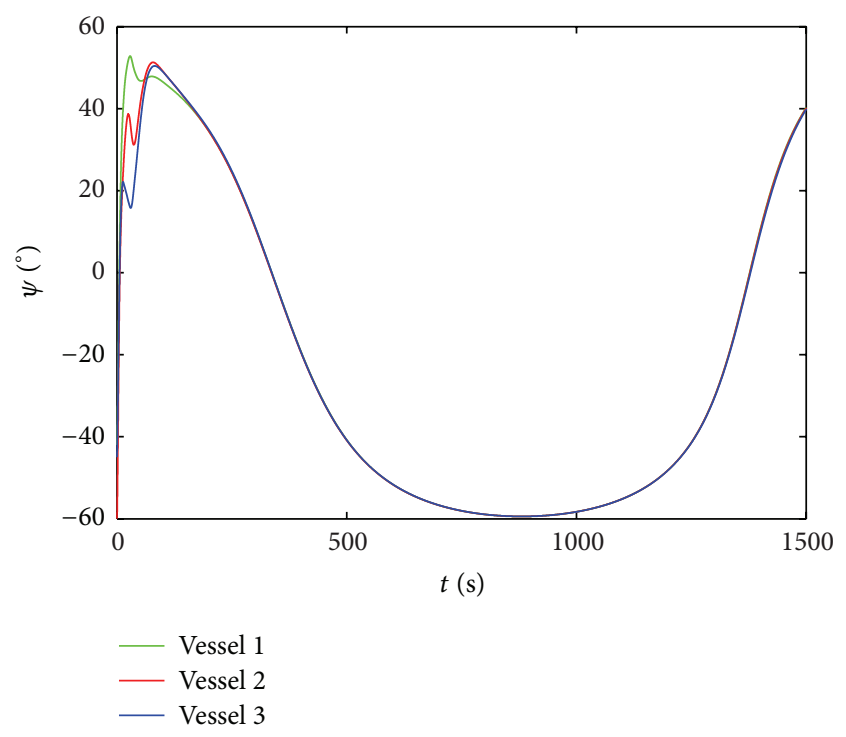

Figure 7: Heading change of the vessels.

position, and heading change curve of the vessel are shown in Figure 3. Figure 4 shows the practical value and estimated value of the surge velocity, sway velocity, and angular velocity of the vessel. The estimated value of the external disturbances are shown in Figure 5. In the simulation experiment, there is no measurement noise in the kinematics and nonlinear dynamics. So the practical value and estimated value of the position and velocity are consensus in a way. What's more, the external disturbances can be estimated through introducing the extended state.

Then we evaluate the effectiveness of the proposed robust coordinated formation control algorithm. Three surface vessels are considered to perform the coordinated tracking task. The initial positions of the three vessels are $\eta_{1}=\left[\begin{array}{lll}65 & 782 & -\pi / 3\end{array}\right]^{T}, \eta_{2}=\left[\begin{array}{lll}80 & 831 & -7 \pi / 30\end{array}\right]^{T}$, and

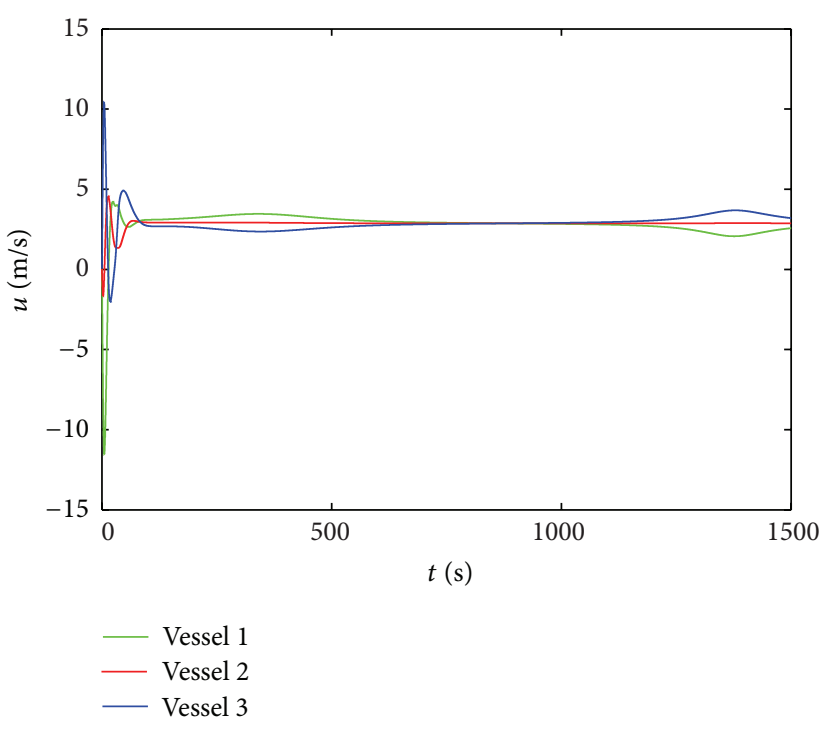

FIGURE 8: Surge velocity change of the vessels.

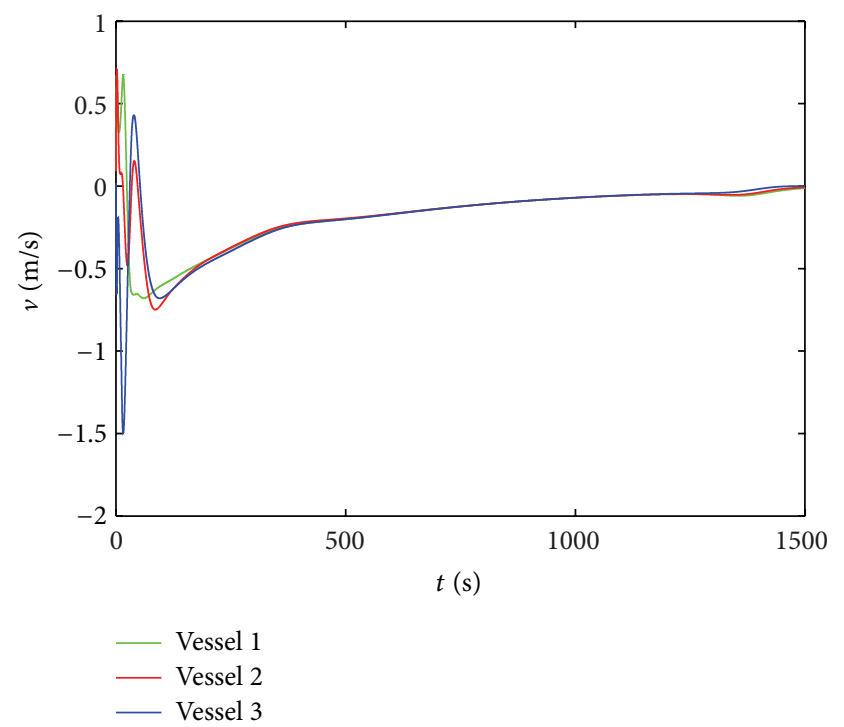

FIGURE 9: Sway velocity change of the vessels.

$\eta_{3}=\left[\begin{array}{lll}75 & 743 & -\pi / 4\end{array}\right]^{T}$, respectively. In order to evaluate the performance of the coordinated tracking, the desired formation pattern of the coordinated formation controller is described by $\mathbf{l}_{1}=\left[\begin{array}{lll}0 & 0 & 0\end{array}\right]^{T}, \mathbf{l}_{2}=\left[\begin{array}{lll}0 & -100 & 0\end{array}\right]^{T}$, and $\mathbf{l}_{3}=\left[\begin{array}{lll}0 & 100 & 0\end{array}\right]^{T}$. The desired trajectory for the assigned leader is chosen as $\eta_{d}(t)=\left[\begin{array}{lll}n_{d} & e_{d} & \psi_{d}\end{array}\right]^{T}$, and the detailed forms are $n_{d}=t, e_{d}=1000 \sin (t / 600), \psi_{d}=\arctan \left(\dot{e}_{d} / \dot{n}_{d}\right)$. The proposed controller parameters are selected as $\Lambda_{1}=$ $\operatorname{diag}(0.05,0.05,0.05), \mathbf{T}=\operatorname{diag}(0.1,0.1,0.1)$, and $\mathbf{K}_{D}=10^{4} *$ $\operatorname{diag}(6.5,6.5,6.5)$.

The simulation results are shown in Figures 6 to 10 . Figure 6 shows the movements for these vessels in the plane. The heading change curve of each vessel is shown in Figure 7. Figures 8,9 , and 10 show the surge velocity, the sway velocity, and the angular velocity of each vessel during the coordinated control process, respectively. We can see that 


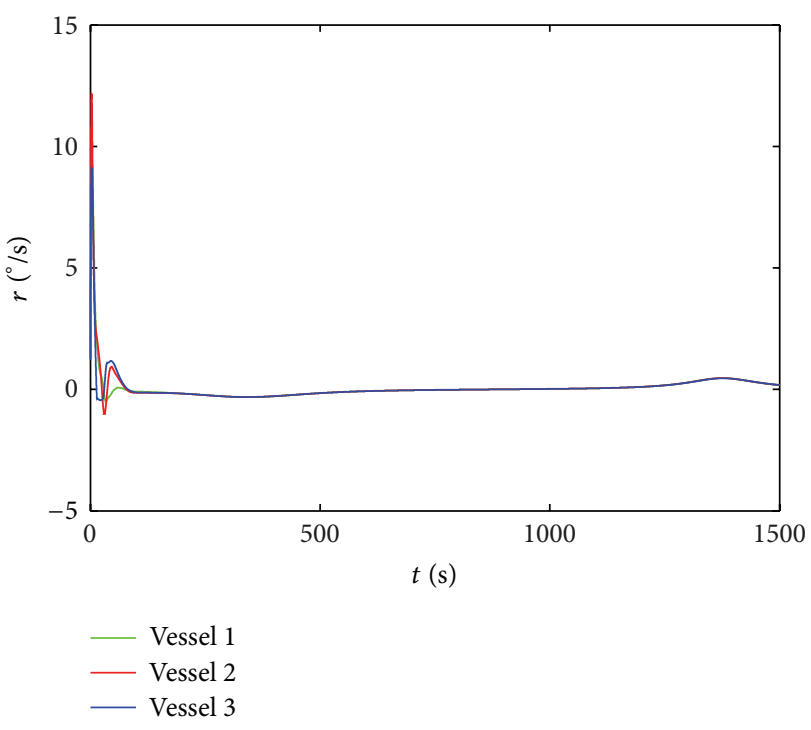

FIgURE 10: Angular velocity change of the vessels.

these vessels realized the coordinated tracking task from Figures 6 and 7. From Figures 8, 9, and 10, the velocities of these vessels achieve consensus as a whole, and the velocities cannot achieve consensus absolutely when the vessels move to the inflexion of the curves. With the analysis of the simulation results, we can conclude that these vessels can accomplish coordinated trajectory tracking task while keeping the desired formation. It means that the proposed coordination control algorithm is effective.

\section{Conclusion}

This paper has proposed a new robust coordinated formation control algorithm for multiple surface vessels in the presence of external environmental disturbances. The proposed coordinated formation controller for these vessels is designed by combining the extended state observer and the dynamic surface control together. The extended state observer is designed to estimate the external disturbances of the surface vessels. The coordinated formation is realized based on the leader-follower strategy. The desired trajectory of each vessel is defined using the desired trajectory of the assigned leader and relative distance with respect to the leader. The controller is designed based on the output of the extended state observer and using the dynamic surface control method. The proposed coordinated controller is robust to the external disturbances. Finally, the effectiveness of the proposed robust coordination control algorithm is demonstrated by the simulation results.

\section{Conflict of Interests}

The authors declare that there is no conflict of interests regarding the publication of this paper.

\section{Acknowledgment}

The authors would like to acknowledge the support of the Natural Science Grant of Liaoning Province (2013020002).

\section{References}

[1] W. Ren, R. W. Beard, and E. M. Atkins, "A survey of consensus problems in multi-agent coordination," in Proceedings of the American Control Conference, pp. 1859-1864, June 2005.

[2] A. Aguiar, J. Almeida, M. Bayat et al., "Cooperative autonomous marine vehicle motion control in the scope of the EU GREX Project: theory and practice," in Proceedings of the IEEE Bremen: Balancing Technology with Future Needs (OCEANS '09 ), pp. 110, May 2009.

[3] P. K. C. Wang, "Navigation strategies for multiple autonomous mobile robots moving in formation," Journal of Robotic Systems, vol. 8, no. 2, pp. 177-195, 1991.

[4] J. K. Hedrick, M. Tomizuka, and P. Varaiya, "Control issues in automated highway systems," IEEE Control Systems Magazine, vol. 14, no. 6, pp. 21-32, 1994.

[5] M. Breivik, V. E. Hovstein, and T. I. Fossen, "Ship formation control: a guided leader-follower approach," in Proceedings of the 17th World Congress, International Federation of Automatic Control (IFAC '08), pp. 16008-16014, July 2008.

[6] G. Antonelli, F. Arrichiello, and S. Chiaverini, "Experiments of formation control with collisions avoidance using the nullspace-based behavioral control," in Proceedings of the 14th Mediterranean Conference on Control and Automation (MED '06), pp. 1-6, June 2006.

[7] J. R. T. Lawton, R. W. Beard, and B. J. Young, "A decentralized approach to formation maneuvers," IEEE Transactions on Robotics and Automation, vol. 19, no. 6, pp. 933-941, 2003.

[8] M. A. Lewis and K.-H. Tan, "High precision formation control of mobile robots using virtual structures," Autonomous Robots, vol. 4, no. 4, pp. 387-403, 1997.

[9] W. Ren and R. W. Beard, "Decentralized scheme for spacecraft formation flying via the virtual structure approach," Journal of Guidance, Control, and Dynamics, vol. 27, no. 1, pp. 73-82, 2004.

[10] J. Almeida, C. Silvestre, and A. M. Pascoal, "Cooperative control of multiple surface vessels with discrete-time periodic communications," International Journal of Robust and Nonlinear Control, vol. 22, no. 4, pp. 398-419, 2012.

[11] C. Thorvaldsen and R. Skjetne, "Formation control of fullyactuated marine vessels using group agreement protocols," in Proceedings the 50th IEEE Conference on Decision and Control and European Control Conference, pp. 4132-4139, 2011.

[12] Y. Wang, W. Yan, and J. Li, "Passivity-based formation control of autonomous underwater vehicles," IET Control Theory \& Applications, vol. 6, no. 4, pp. 518-525, 2012.

[13] M. Fu and J. Jiao, "A hybrid approach for coordinated formation control of multiple surface vessels," Mathematical Problems in Engineering, vol. 2013, Article ID 794284, 8 pages, 2013.

[14] I.-A. F. Ihle, J. Jouffroy, and T. I. Fossen, "Formation control of marine surface craft: a lagrangian approach," IEEE Journal of Oceanic Engineering, vol. 31, no. 4, pp. 922-934, 2006.

[15] F. Fahimi, "Non-linear model predictive formation control for groups of autonomous surface vessels," International Journal of Control, vol. 80, no. 8, pp. 1248-1259, 2007.

[16] J. Almeida, C. Silvestre, and A. Pascoal, "Cooperative control of multiple surface vessels in the presence of ocean currents and parametric model uncertainty," International Journal of Robust and Nonlinear Control, vol. 20, no. 14, pp. 1549-1565, 2010.

[17] F. Fahimi, "Sliding-mode formation control for underactuated surface vessels," IEEE Transactions on Robotics, vol. 23, no. 3, pp. 617-622, 2007. 
[18] S. Yin, S. Ding, and H. Luo, "Real-time implementation of fault tolerant control system with performance optimization," in Proceedings of the IEEE Transactions on Industrial Electronics, vol. 61, no. 5, 2013.

[19] S. Yin, S. Ding, A. Haghani, H. Hao, and P. Zhang, "A comparison study of basic data-driven fault diagnosis and process monitoring methods on the benchmark Tennessee Eastman process," Journal of Process Control, vol. 22, no. 9, pp. 1567-1581, 2012.

[20] S. Yin, S. X. Ding, A. H. A. Sari, and H. Hao, "Data-driven monitoring for stochastic systems and its application on batch process," International Journal of Systems Science, vol. 44, no. 7, pp. 1366-1376, 2013.

[21] E. Børhaug, A. Pavlov, E. Panteley, and K. Y. Pettersen, "Straight line path following for formations of underactuated marine surface vessels," IEEE Transactions on Control Systems Technology, vol. 19, no. 3, pp. 493-506, 2011.

[22] J. Ghommam and F. Mnif, "Coordinated path-following control for a group of underactuated surface vessels," IEEE Transactions on Industrial Electronics, vol. 56, no. 10, pp. 3951-3963, 2009.

[23] X. P. Shi, S. R. Liu, and F. Liu, "New robust control strategy for module manipulators via sliding mode control with an extended state observer," Proceedings of the Institution of Mechanical Engineers I, vol. 224, no. 5, pp. 545-555, 2010.

[24] Y. Xia, Z. Zhu, and M. Fu, "Back-stepping sliding mode control for missile systems based on an extended state observer," IET Control Theory \& Applications, vol. 5, no. 1, pp. 93-102, 2011.

[25] S. E. Talole, J. P. Kolhe, and S. B. Phadke, "Extended-stateobserver-based control of flexible-joint system with experimental validation," IEEE Transactions on Industrial Electronics, vol. 57, no. 4, pp. 1411-1419, 2010.

[26] M. Fu, J. Jiao, and L. Hao, "A coordinated dyanmic positioning control algorithm based on active disturbance rejection control," in Proceedings of IEEE International Joint Conference on Computational Sciences and Optimization, pp. 67-71, 2012.

[27] B.-Z. Guo and Z.-1. Zhao, "On the convergence of an extended state observer for nonlinear systems with uncertainty," Systems \& Control Letters, vol. 60, no. 6, pp. 420-430, 2011.

[28] B.-Z. Guo and Z.-L. Zhao, "On convergence of non-linear extended state observer for multi-input multi-output systems with uncertainty," IET Control Theory \& Applications, vol. 6, no. 15, pp. 2375-2386, 2012.

[29] B.-Z. Guo and Z.-L. Zhao, "On convergence of the nonlinear active disturbance rejection control for MIMO systems," SIAM Journal on Control and Optimization, vol. 51, no. 2, pp. 1727-1757, 2013.

[30] T. Fossen, Marine Control Systems: Guidance, Navigation and Control of Ships, Rigs and Underwater Vehicles, Marine Cybernetics, Trondheim, Norway, 2002. 


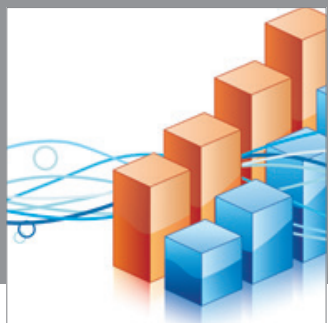

Advances in

Operations Research

mansans

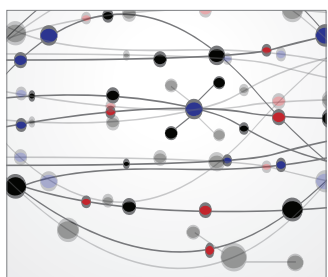

The Scientific World Journal
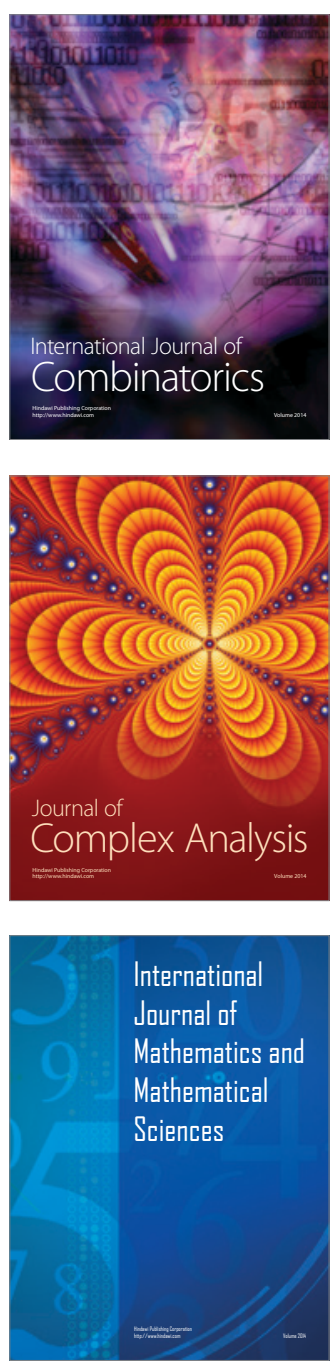
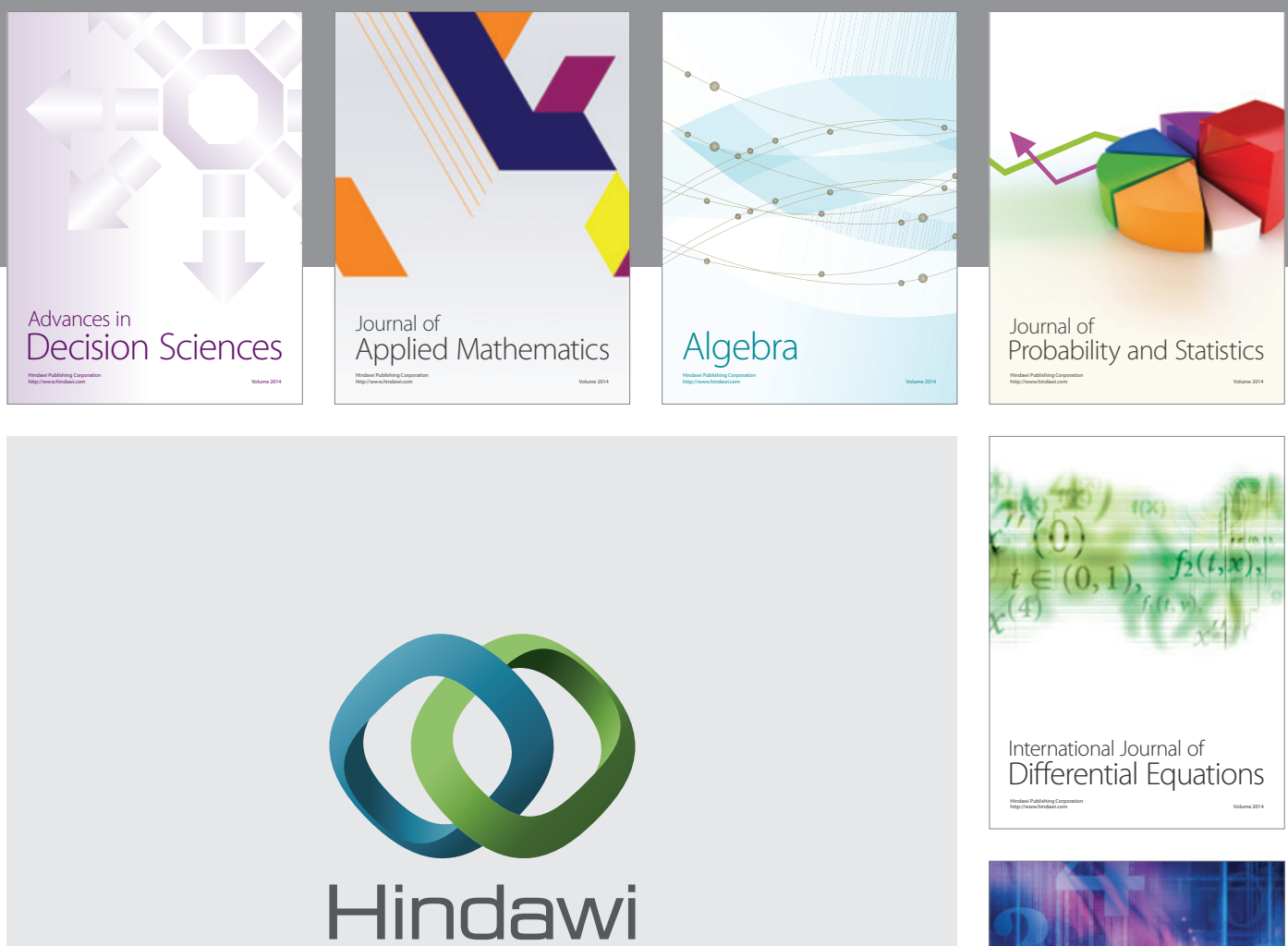

Submit your manuscripts at http://www.hindawi.com
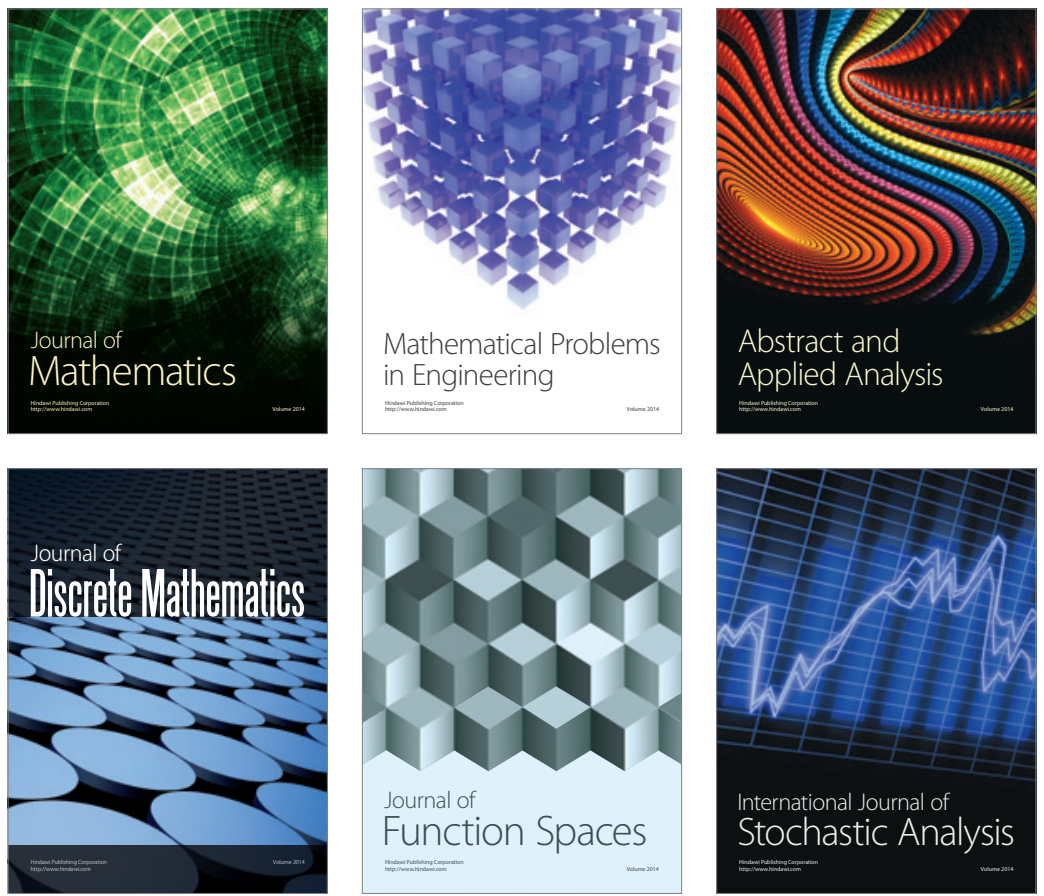

Journal of

Function Spaces

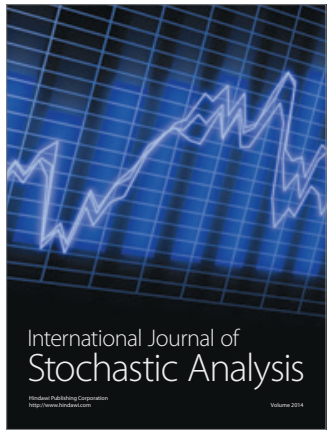

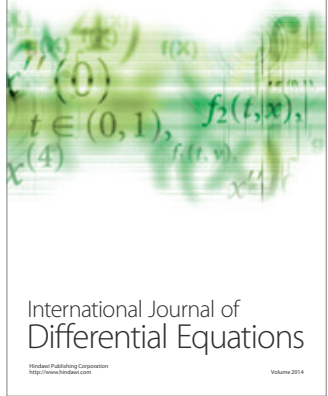
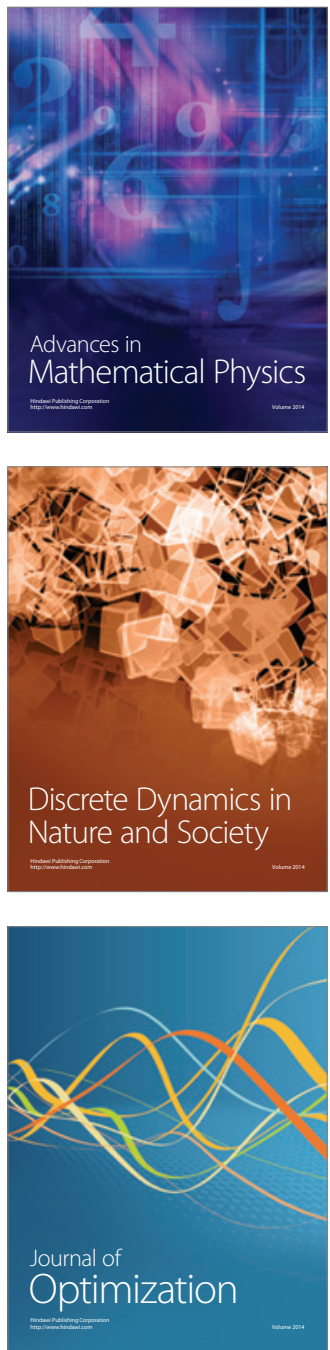